\title{
Transcranial Photobiomodulation Therapy in the Cognitive Rehabilitation of Patients with Cranioencephalic Trauma
}

\author{
Ana Maria Costa Carneiro, PhD, ${ }^{1}$ Guilherme Cruz Poiani, MS, ${ }^{2}$ Ana Luiza Zaninnoto, PhD, ${ }^{3}$ \\ Rodrigo Lazo Osorio, PhD, ${ }^{1,4}$ Marcelo de Lima Oliveira, MD, \\ Wellingson Silva Paiva, MD, PhD, ${ }^{2}$ and Renato Amaro Zângaro, PhD, Postdoc ${ }^{1,4}$
}

\begin{abstract}
Objective: This research evaluated the hemodynamic conditions before and after the transcranial photobiomodulation therapy (PBMT) and investigated neurocognitive changes before and after treatment.

Background: Traumatic brain injury (TBI) is the major cause of morbidity and mortality among individuals 21-60 years old and causes $\sim 500,000$ people to be hospitalized in Brazil annually. Some survivors develop an irreversible decrease in neurological function, and the mortality rate is as high as $70 \%$ in severe cases. $P B M T$ is an alternative to treat secondary injuries due to TBI.

Methods: This multidisciplinary clinical study was carried out on 10 chronic adult patients with severe TBI, who were treated with $P B M T$ with an optical device containing 13 sets of 4 light emitting diodes, and underwent hemodynamic transcranial Doppler and neuropsychological evaluation at three different times: pre-PBMT, postPBMT (after a week), and late-PBMT, which occurred 3 months after the last session. The patients received PBMTs three times a week, for 6 weeks. PBMTs were performed for 18 sessions for 6 weeks and 30 min per session.

Results: The results found an alteration in the cerebral blood flow (CBF) as well as a consequent increase of the cerebral oxygenation that helped to improve the cerebral function.

Conclusions: The PBMT contributed to increased CBF, evidenced mainly by the increased left peak systolic velocity, which consequently increased the hemodynamic response after the PBMT and impacts on the peripheral cerebral perfusion contributing to improved cerebral function.
\end{abstract}

Keywords: photobiomodulation, traumatic brain injury, neurological rehabilitation

\section{Introduction}

$\mathbf{T}$ RAUMATIC BRAIN INJURY (TBI) is the leading cause of worldwide morbidity and mortality among individuals between the age of 21 and 60 years, with about 500,000 people hospitalized annually in Brazil. This injury, generated by external trauma, causes anatomical and functional changes in the brain. It might also disrupt the axonal and microvasculature, resulting in diffuse axonal injury (DAI). ${ }^{1-3}$
It is estimated that about one-third of LAD victims die. Commonly, survivors have persistent neuropsychological deficits, and impaired consciousness is more frequent than in other types of closed skull trauma. ${ }^{4,5}$

Low-power light therapy, as also known as photobiomodulation therapy (PBMT), has been investigated, and evidence suggests that the interaction of light with biological tissues can obtain excellent therapeutic results. ${ }^{6}$ Photobiomodulation $(\mathrm{PBM})$ is an incoherent radiation source

\footnotetext{
${ }^{1}$ Stricto Sensu Program Master and Doctorate in Biomedical Engineering, Anhembi Morumbi University, São José dos Campos, São Paulo, Brasil.

${ }^{2}$ Division of Neurosurgery, Hospital das Clínicas of the University of São Paulo Medical School (HCFMUSP), São Paulo, Brasil.

${ }^{3}$ Speech and Feeding Disorders Lab, MGH Institute of Health Professional, Marion, Ohio.

${ }^{4}$ Center for Innovation Technology and Education (CITÉ), Anhembi Morumbi University, São José dos Campos, São Paulo, Brasil.

(C) Ana Maria Costa Carneiro, et al., 2019; Published by Mary Ann Liebert, Inc. This Open Access article is distributed under the terms of the Creative Commons Attribution Noncommercial License (http://creativecommons.org/licenses/by-nc/4.0/) which permits any noncommercial use, distribution, and reproduction in any medium, provided the original author(s) and the source are credited.
} 
with great divergence of light beam and high optical output power. Strong evidence exists of the therapeutic effects of the PBM to treat pain and other symptoms. ${ }^{7-11}$ It is painless and quite efficient in several therapeutic modalities, with antiedematous and analgesic effects. PBM stimulates the release of endorphins, inhibits nociceptor signals, controls the mediators of pain, causes anti-inflammatory effects, reduces tissue edema and hyperemia, increases vascularity, restores neural function after injury, and modulates immune system cells to assist in the repair process. ${ }^{12-15}$

This study examined a noninvasive method of neural stimulation to safely penetrate the brain and increase cell metabolism during treatment, and studied whether these effects may persist after the end of treatment. ${ }^{11,13-15}$ Based on these principals, this study aimed to evaluate hemodynamic conditions before and after the PBMT to verify the possible mediation and late neurocognitive alteration of patients attended at the Neurotraumatic Outpatient Clinic of the Neurosurgery Division-Clinical Hospital of the Faculty of Medicine at the University of São Paulo, Brazil (HCFMUSP).

\section{Methods}

This is a multidisciplinary clinical study. Therefore, 14 patients, who had suffered TBI and were between 18 and 60 years old, were selected. The participants were an average age of $37.8 \pm 10.2$ years and of both genders. The time after TBI ranged from 4 months to 4 years. Later, 1 participant dropped out and 3 were excluded because they had not performed transcranial Doppler (TD) before and after the PBMT; thus, 10 participants remained in the study. They presented different types of lesions, among them subdural hematoma, subarachnoid hemorrhage, diffuse axonal lesion, and contusions. Participants were evaluated each time, keeping the same order, and all of them achieved the maximum score (15) of the Glasgow Coma Scale and underwent TD before and after PBMT and received three neuropsychological evaluations: pre-, post-PBMT (after a week), and late-PBMT (3 months after the last session; Fig. 1).

The epidemiological and clinical characteristics of the sample are described in Table 1.

The project was approved by the Research Ethics Committee of the Hospital of Clinics of the Faculty of Medicine of the University of São Paulo (CAPPESQ-HCFMUSP) and the Research Ethics Committee of HCFMUSP and approved by the CAAE under number: 33563014.2.0000.0068, respecting all ethical and legal precepts established by resolution 466/2012.

\section{Procedures}

The participants' cerebral blood flow $(\mathrm{CBF})$ with DT were evaluated at two times: pre- and post-transcranial photobiomodulation. Evaluation occurred after a week of PBMT.

\section{Protocol for TD}

The equipment used for the evaluation was Doppler (Multidop X-4; DWL, Sipplingen, Germany), operating in the frequency of $7.5 \mathrm{MHz}$ in B-mode with linear probe. It rated the arteries of the polygon of Willis, the peak systolic velocity (PSV), the end diastolic flow velocity (DFV), and the pulsatility index (PI). The focus was to evaluate the right and left middle cerebral arteries (MCAR and MCAL), because of the easy access by transtemporal acoustic pathways (located above the zygomatic arch) due to its fine bone structure. ${ }^{16}$

The most valuable findings in the TD interpretation were the $\mathrm{CBF}$ and the PI, which were described by the degree of variability between the highest blood flow speed that occurred during the different phases of the cardiac cycle (systole and diastole), consequently demonstrating the peripheral resistance of the organ supplied by the analyzed artery.

The examination was performed at the HCFMUSPDivision of Neurosurgery.

\section{Neuropsychological evaluation protocol}

The neuropsychological evaluation determined emotional and cognitive measures, as well as characterized their complexity and severity that are directly related to the type of brain injury.

\section{Neuropsychological tests}

Each assessment session lasted $\sim 1 \mathrm{~h}$ and $30 \mathrm{~min}$.

Patients completed the questionnaires either independently or with the help of the neuropsychologist:

The Beck depression inventory (BDI)-II is a structured instrument with 21 categories of depressive symptoms and attitudes that allow self-description of the cognitive behavioral and somatic manifestations of depression. Higher scores indicate high levels of depressive symptoms.

The Beck anxiety inventory (BAI) is a structured instrument with 21 multiple-choice questions, which is used for self-assessment of anxiety levels. Higher scores indicate high levels of anxiety.

The Stroop test-Version Victoria (selective inhibition and attention) evaluates the speed of information processing,

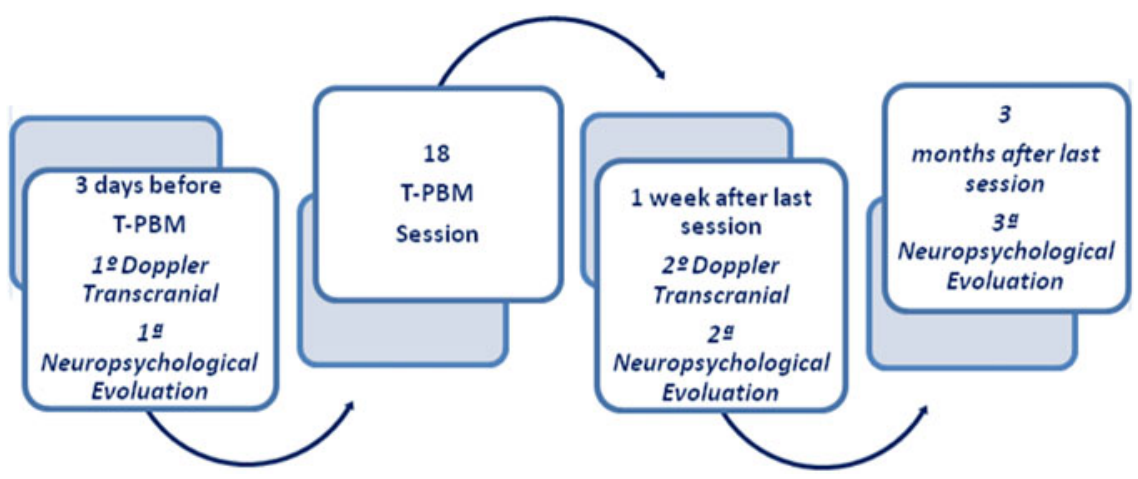

FIG. 1. Transcranial photobiomodulation protocol. 
Table 1. Epidemiological and Clinical CHARACTERISTICS

\begin{tabular}{lc}
\hline Epidemiological and clinical characteristics & $\begin{array}{c}\text { General } \\
\mathrm{n}=10(\%)\end{array}$ \\
\hline Male & $9(90.0)$ \\
Age (standard deviation and mean) & $37.8 \pm 10.2$ \\
Years of studies & \\
$\quad$ Up to 8 years & $4(40.0)$ \\
From 8 to 11 years & $1(10.0)$ \\
From 11 to 15 years & $5(50.0)$ \\
Glasgow coma scale & \\
3T* & $8(80.0)$ \\
3 & $1(10.0)$ \\
6 & $1(10.0)$ \\
Mechanism of trauma & \\
Automobile accident & $6(60.0)$ \\
High plane drop & $3(30.0)$ \\
Hit run over & $1(10.0)$ \\
\hline
\end{tabular}

3T*, classification of GCS in intubated patients.

selective attention, and executive function of inhibition and control. In the test, three cards are presented to the participants at different times evaluating their ability to differentiate colors and words. The score is measured in seconds of execution.

Trail making test-forms A and B (TMT A and TMT B) both require perceptual tracking of a sequence and speed performance; TMT B also requires divided attention. The score is measured in seconds of execution.

The symbol digit test (processing speed) is applied to evaluate processing speed. The participant must be able to relate a symbol to a digit as fast as possible. The score is measured in seconds of execution.

The Rey auditory verbal learning test (RAVLT—episodic memory) assesses episodic, immediate memory, verbal learning, susceptibility to interference (proactive and retroactive), retention of information, and recognition. The scores are computed according to the number of correctly recalled words.

The complex Rey figure (visual construction and memory) is used to evaluate visual memory, visuospatial ability, and some functions of planning. Individuals first copy the complex Rey figure and after $3 \mathrm{~min}$, they are asked to reproduce the same complex figure. Higher scores indicate better performance. ${ }^{17-19}$

The verbal fluency (phonological F-A-S, and semantic category). For the phonological test, participants must recall as many words as possible beginning with the letters F-A-S with $60 \mathrm{sec}$ for each word. For the semantic category test, the participants recall as many words as possible related to a specific categorical group (animals or fruits) for $60 \mathrm{sec}^{20}$

\section{PBMT protocol}

Participants were presented the research protocol, and the optical device was demonstrated. After receiving information, the participants signed the informed consent term (ICT) and initiated the PBMT protocol.

The radiation source for the applied $P B M T$ was an optical device containing 13 sets of 4 light emitting diodes (LEDs), evenly distributed over the skull cap with a mean area of
$400 \mathrm{~cm}^{2}$. The model device PBMT 1 -Medical SystemsSão José dos Campos emits radiation in the red range with peak at $630 \mathrm{~nm}$.

\section{Calculation of irradiated area}

To calculate the radiated area of the skull, the equation of an ellipsoid of revolution was used, which in this case is scalene $(\mathrm{a} \neq \mathrm{b} \neq \mathrm{c})$, where " $p$ " is a constant with a value of $\sim 1.6075$;

$$
\approx 4 \pi\left(\frac{a^{p} b^{p}+a^{p} c^{p}+b^{p} c^{p}}{3}\right) 1 / p,
$$

in which $\mathrm{a}=10.5 \mathrm{~cm}$ in length (long axis), $\mathrm{b}=8.5 \mathrm{~cm}$ in length (shortest axis), and $\mathrm{c}=5.0 \mathrm{~cm}$ in length (distance from the focus to the center of the ellipse). Applying the above values gives an area of the cranial box equal to $400 \mathrm{~cm}^{2}$.

\section{Calculation of energy density (fluency)}

To calculate the applied energy density, Equation 2 was used:

$$
D E\left(J / \mathrm{cm}^{2}\right)=\frac{\text { power } \times \text { time }}{\text { area }} . \quad E q .2
$$

The set of LEDs contained in the optical device has a total optical power of $830 \mathrm{~mW}$, which after crossing the cerebral structure reduces to $25.73 \mathrm{~mW} / \mathrm{cm}^{2}$; thus, $0.12 \mathrm{~J} / \mathrm{cm}^{2}$ is expected to reach the gray mass, the layer more external, formed by the bodies of neurons. Considering the irradiation time equal to $30 \mathrm{~min}$, we can calculate the energy density, which in this case is equal to $3.74 \mathrm{~J} / \mathrm{cm}^{2}$ and a total of $1800 \mathrm{sec}$.

The $P B M T$ protocol was performed three times a week for 6 weeks, totaling 18 sessions and a total of $32,400 \mathrm{sec}$ of irradiation, according to Table 2 .

\section{Statistical analysis}

Descriptive analysis of the results considered the quantitative variables represented by their average and standard deviations when their distributions were normal, and by medians and interquartiles when not normal. The definition of normality was made through graphical analysis and Shapiro-Wilk test. The variables were represented by frequencies and percentages.

To evaluate the TD results, data were grouped and compared as the main dependent variable using the variation of the mean flow velocity (MFV) in the middle cerebral artery (MCA); therefore, a parametric analysis paired with the Student's $t$-test was used, as explained in the results. The results were considered significant when $p$ values $<0.05$. The analyses were performed using the IBM Statistical Package for Social Sciences (SPSS ${ }^{\circledR}$, Chicago, IL) 20.0.

\section{Results}

In this study, the evaluation of cerebral hemodynamic conditions was intended to identify possible alterations in the flow of MCAR and MCAL due to the high degree of 
Table 2. Parameters in Experimental and Clinical Transcranial Photobiomodulation Articles

\section{Manufacturer}

Model identifier

Year produced

Number and type of emitters (laser or LED)

Wavelength and bandwidth [nm]

Pulse mode [CW or $\mathrm{Hz}$, duty cycle]

Power density at target $\left[\mathrm{mW} / \mathrm{cm}^{2}\right]$

Exposure duration [sec]

Energy density $\left[\mathrm{J} / \mathrm{cm}^{2}\right]$

Radiant energy [J]

Number of points irradiated

Area irradiated $\left[\mathrm{cm}^{2}\right]$

Number and frequency of treatment sessions

Total radiant energy over entire treatment course $[\mathrm{J}]$

LED, light emitting diode; T-PBM, transcranial photobiomodulation. impairment by the TBI, according to the lesioned area. The CBF of the MCAR and MCAL, the PSV, and the end diastolic velocity (EDV) were measured to verify the CBF changes.

The most frequent diagnosis was DAI, usually associated with other types of lesions, and in this study, four participants were affected by this lesion according to Fig. 2A. Subarachnoid hemorrhage was present in three participants (Fig. 2B), two presented subdural hematoma, and contusions were less frequent and more diverse (Fig. 2C), including a right frontal bruise and another temporal parietal associated with other lesions (Fig. 2D).

The correlation between the type of lesion, the period between the occurrence of TBI and the beginning of the PBMT, and the results of the average velocity in the right and left cerebral arteries pre- and post-PBMT are described in Table 3.

Therapeutic responses are most likely to depend on the length of time between the occurrence of TBI and the beginning of $P B M T$. In this study, all subjects who underwent $P B M T$ had suffered a TBI for at least 4 months and a maximum of 4 years before $P B M T$.

From the use of the TD technique, Table 4 compares the pre- and post-PBMT results of MFV, MCAD, and MCAL considering the different types of trauma and the time lag between trauma and therapy.

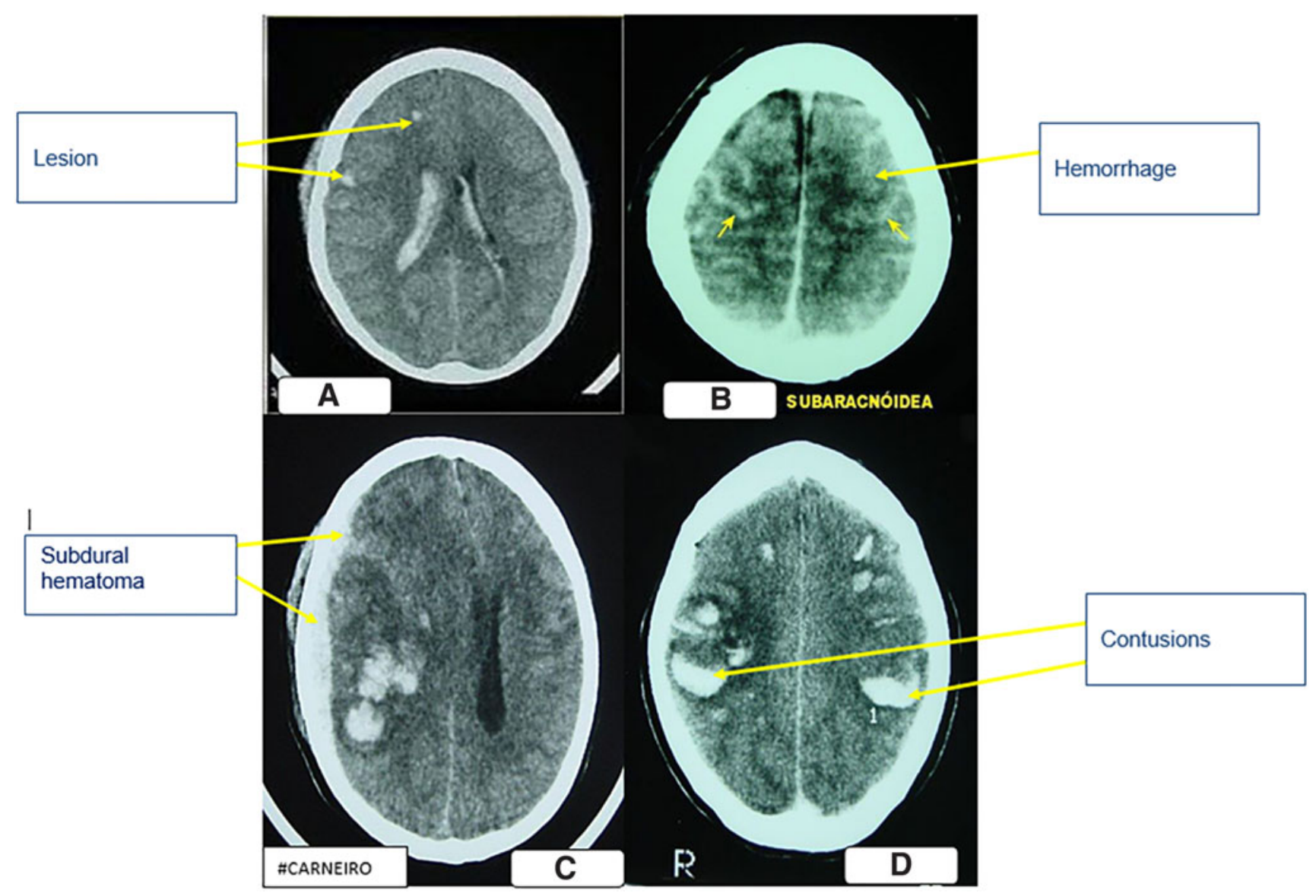

FIG. 2. (A) Tomographic image of diffuse axonal lesion. (B) Tomographic image of subarachnoid hemorrhage with striae hyperdens. (C) Tomographic image of acute subdural hematoma, with swelling of the left hemisphere. (D) Tomographic image of bilateral brain contusions. http://anatpat.unicamp.br/radhsa1.html. 
Table 3. Correlation Between Injured Area and Mean Flow Velocity of Middle Cerebral Arteries Right and Middle Cerebral Arteries Left Pre- and Post-Transcranial Photobiomodulation

\begin{tabular}{|c|c|c|c|c|c|c|}
\hline Participant & Local/type of injury & $\begin{array}{l}\text { Time } \\
\text { of } \\
\text { injury }\end{array}$ & $\begin{array}{c}M F V- \\
M C A R \\
\text { pre- } T P B M \\
\mathrm{~cm} / \mathrm{s}\end{array}$ & $\begin{array}{c}M F V- \\
M C A R \\
\text { post }-T-P B M \\
\mathrm{~cm} / \mathrm{s}\end{array}$ & $\begin{array}{c}M F V- \\
M C A L \\
\text { pre- } T-P B M \\
\mathrm{~cm} / \mathrm{s}\end{array}$ & $\begin{array}{c}M F V- \\
M C A L \\
\text { post }-T-P B M \\
\mathrm{~cm} / \mathrm{s}\end{array}$ \\
\hline 1 & Frontal subgaleal hematoma DAI & 4 months & 55.4 & 56.9 & 61.7 & 61.8 \\
\hline 2 & DAI & 8 months & 34.2 & 45 & 44.3 & 59 \\
\hline 3 & TBI with DAI. Frontal Petechiae & 7 months & 42 & 44 & 44 & 45 \\
\hline 4 & DAI & 18 months & 56 & 42 & 65 & 58 \\
\hline 5 & $\begin{array}{l}\text { Right temporal contusion and right frontal } \\
\text { contusion }\end{array}$ & 24 months & 48 & 30 & 31 & 39 \\
\hline 6 & $\begin{array}{l}\text { Left temporal parietal contusion } 4 \mathrm{~cm} \text {, } \\
\text { frontal bruising on the right } \\
\text { subarachnoid hemorrhage** }\end{array}$ & 48 months & 37 & 51 & 31 & 34 \\
\hline 7 & $\begin{array}{l}\text { Subarachnoid hemorrhage, diffuse } \\
\text { cerebral swelling, left subgaleal } \\
\text { hematoma }\end{array}$ & 7 months & 48.9 & 56.3 & 48.1 & 57.9 \\
\hline 8 & $\begin{array}{l}\text { Subarachnoid hemorrhage ventricular } \\
\text { hemorrhage, bifrontal fracture }\end{array}$ & 4 months & 57.5 & 32 & 49.5 & 49 \\
\hline 9 & Right anterior frontal subdural collection & 24 months & 28 & 30 & 38 & 30 \\
\hline 10 & $\begin{array}{l}\text { Acute subdural hematoma, left } \\
\text { hemisphere cerebral swelling }\end{array}$ & 36 months & 41 & 42 & 40 & 42 \\
\hline
\end{tabular}

Bold values indicate increase of Vm of the MCAR and MCAL.

Healthy people present MFV MCAR and MCAL between 42 and $82 \mathrm{~cm} / \mathrm{sec}^{21}$

Italic values lie outside this range.

Bold italic values indicate an increase of the MFV MCAR and MCAL, however, outside the normal range.

**Participant framed in two different types of injuries.

DAI, diffuse axonal injury; MCAL, middle cerebral arteries left; MCAR, middle cerebral arteries right; MFV, mean flow velocity; TBI, traumatic brain injury.

The MFV, MCAD, and MCAL results presented in Table 3 indicate that the participants affected by DAI up to 8 months had better responses to the PBMT than those with other types of lesions, even if these results were not statistically significant.

The following histogram presents $\mathrm{CBF}$ results in MCAD and MCAL at the pre- and post-PBMT tests, with an increase in mean CBF in MCAL post-PBMT, although without statistical significance. Figure 3 shows the results of bilateral PSV before and after PBMT. The averages were elevated in post- $P B M T$ in both hemispheres, and the left hemisphere PSV presented a significant increase $(p<0.007)$.

The TD results in the pre- and post-PBMT periods are observed in Table 4, with the main indicators of blood flow in MCAR and MCAL.

The hemodynamic evaluation of MCAR found a discrete reduction of $\mathrm{CBF}$, but with a relative increase of PSV and end diastolic velocity (EDV), although without significance. The PI measures the variation of the highest flow velocity that occurs during the different phases of the cardiac cycle, expressing the peripheral resistance of the organ supplied by the analyzed artery. The established parameters for PI range from 0.49 to 1.01 ; the average PI pre-PBMT was 1.05 and that post-PBMT was reduced to 1.02 .

\section{Neuropsychological evaluation}

The results of neuropsychological evaluations indicate an improvement in the performance of some test that evaluated brain function. Table 5 presents the results of neuropsychological performance comparing pre-PBMT, post-PBMT, and late-PBMT. In some tests, higher scores indicate better results (complex Rey figure; RAVLT, verbal fluency test F-A-S, and

Table 4. Evaluation of Transcranial Doppler Pre- and Post-Transcranial Photobiomodulation

\begin{tabular}{lccr}
\hline Characteristic & Preintervention & Postintervention & Variation \\
\hline Right side & & & $\mathrm{p}$ \\
PI & $1.05 \pm 0.34$ & $1.02 \pm 0.39$ & $0.03 \pm 0.43$ \\
PSV & $66.9 \pm 19.0$ & $68.6 \pm 13.7$ & $-1.7 \pm 21.0$ \\
Final DFV & $27.3 \pm 11.6$ & $30.5 \pm 8.9$ & $-3.2 \pm 15.4$ \\
MCA MFV & $44.8 \pm 10.0$ & $42.9 \pm 10.0$ & $1.9 \pm 13.0$ \\
Left side & & & 0.842 \\
PI & $0.79 \pm 0.21$ & $0.96 \pm 0.28$ & 0.528 \\
PSV & $65.7 \pm 17.1 *$ & $78.0 \pm 12.3 *$ & $-0.17 \pm 0.32$ \\
Final DFV & $35.7 \pm 12.2$ & $36.8 \pm 5.6$ & $-1.1 \pm 11.1$ \\
MCA MFV & $45.3 \pm 11.4$ & $47.6 \pm 11.3$ & $-2.3 \pm 7.1$ \\
\hline
\end{tabular}

${ }^{*} p<0.05$.

DFV, diastolic flow velocity; MCA, middle cerebral artery; PI, pulsatility index; PSV, peak systolic velocity. 


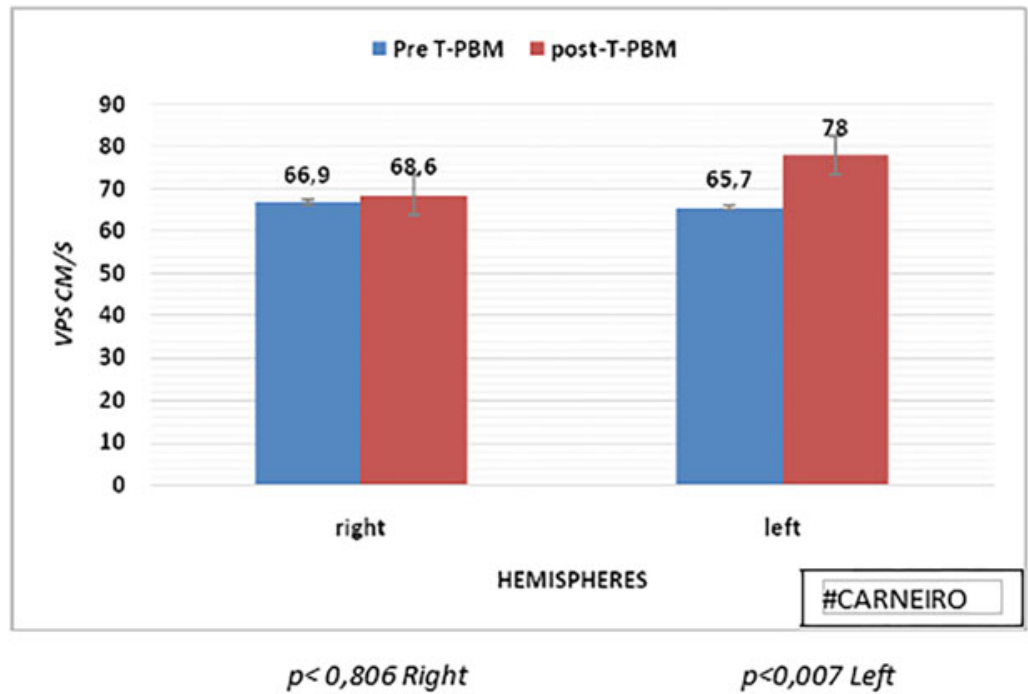

FIG. 3. Histogram of comparative PSV of right and left hemispheres, pre- and postPBM. PSV, peak systolic velocity.

semantic); but for tests that evaluated the response time (symbol digit, Stroop test, TMT A, and TMT B), better results were for faster execution time.

Thus, the Rey's figure test found improvement in relation to the pre-PBMT evolution at both times: post-PBMT and late-PBMT. In the evaluation of TMT A and TMT B, the response time reduced at both times. The Rey's complex figure-immediate recall found better performance in the post-PBMT than in the late-PBMT $(p<0.271)$. In the postinterference recall, Rey's figure performance was also better in late-PBMT evaluation, as well as in E7 recall.

Post-PBMT, response time increased in the Stroop 1 and 3 tests; thus, the performance was lower than the pre-PBMT evaluation. Stroop 2 presented better performance in the post-PBMT evaluation with an increased time in the late$P B M T$ evaluation.

In the F-A-S evaluation, the performance improved in the post-PBMT evaluation and decreased in the late-PBMT evaluation. This performance was repeated in the semantic F-A-S.

The evaluation of Rey's figure-immediate recall found an improvement in the average performance between the pre$P B M T$ and post-PBMT mediate tests.

In the RAVLT evaluation, individual performance increased for some study participants, with increased performance identified between pre-PBMT, post-PBMT, and latePBMT

The performance evaluation in the Stroop test showed small variations between the pre-PBMT, post-PBMT, and late-PBMT evaluations, also not presenting statistical significance for the evaluation of the selective functions, processing speed, and construction of the executive function called "inhibition" and "control" evaluated by this test.

Table 6 presents the results of the variation of the selfassessment of BDI-II and BAI. The BDI evaluation demonstrated a reduction of the average scores in the post-PBMT

Table 5. Neuropsychological Evaluation Pre-Transcranial Photobiomodulation, Post-Transcranial Photobiomodulation and Late-Transcranial Photobiomodulation

\begin{tabular}{lcccc}
\hline Characteristic & Rating & Pre-T-PBM & Post-T-PBM & Late-T-PBM \\
\hline F. Rey & $\mathbf{\Delta}$ & $25.4 \pm 9.9$ & $26.1 \pm 9.2$ & $26.9 \pm 6.7$ \\
TMT A & $\mathbf{\nabla}$ & $75.6 \pm 29.3$ & $72.8 \pm 50.5$ & $68.4 \pm 37.6$ \\
TMT B & $\mathbf{\Delta}$ & $247.1 \pm 228.7$ & $186.6 \pm 153.8$ & $153.6 \pm 84.9$ \\
Rey's. figure Immediate recall & $\mathbf{\Delta}$ & $11.1 \pm 8.1$ & $12.9 \pm 7.9$ & $12.3 \pm 8.9$ \\
RAVLT (E1-E55) & $\mathbf{\Delta}$ & $34.0 \pm 9.4$ & $36.4 \pm 5.9$ & $37.7 \pm 7.4$ \\
Recall postinterference (E6) & $\mathbf{\Delta}$ & $5.1 \pm 3.8$ & $5.9 \pm 2.5$ & $6.1 \pm 3.2$ \\
Recall (E7) & $\mathbf{\nabla}$ & $12.7 \pm 2.6$ & $5.6 \pm 2.7$ & $6.4 \pm 3.4$ \\
Recognition & $\mathbf{\nabla}$ & $23.6 \pm 4.0$ & $11.3 \pm 2.7$ & $11.6 \pm 5.3 *$ \\
Stroop 1 & $\mathbf{\nabla}$ & $28.1 \pm 7.0$ & $25.7 \pm 12.3$ & $23.0 \pm 6.2$ \\
Stroop 2 & $\mathbf{\Delta}$ & $39.1 \pm 10.3$ & $27.1 \pm 10.5$ & $30.0 \pm 9.8 *$ \\
Stroop 3 & $\mathbf{\Delta}$ & $12.7 \pm 9.5$ & $39.9 \pm 24.0$ & $38.4 \pm 10.9$ \\
F-A-S & $\mathbf{\Delta}$ & $29.4 \pm 8.9$ & $14.0 \pm 4.6$ & $27.6 \pm 8.5$ \\
Semantic F-A-S & $\mathbf{\Delta}$ & $5.5 \pm 2.9$ & $29.9 \pm 9.8$ & $13.0 \pm 3.6$ \\
Symbol & $\mathbf{\Delta}$ & $10.3 \pm 4.1$ & $5.5 \pm 3.2$ & $31.3 \pm 13.2$ \\
SNL & & & $11.2 \pm 4.3$ & $5.0 \pm 3.6$ \\
Dígits & & & $11.4 \pm 3.4$ \\
\hline
\end{tabular}

$\boldsymbol{\Delta}$, better performance at higher scores.

$\boldsymbol{\nabla}$, better performance at lower scores.

* Refers to the two tests that did not perform well in cognitive assessment. 
Table 6. Assessment of Anxiety and Depression Pre-Transcranial Photobiomodulation, Post-Transcranial Photobiomodulation, and Late-Transcranial Photobiomodulation

\begin{tabular}{lccccc}
\hline Characteristic & Rating & $\begin{array}{c}\text { Pre-T-PBM and post-T-PBM } \\
\text { variation mediate }\end{array}$ & $\mathrm{p}$ & $\begin{array}{c}\text { Pre-T-PBM and post-T-PBM } \\
\text { variation late }\end{array}$ & $\mathrm{p}$ \\
\hline BDI & $\mathbf{V}$ & $3.1 \pm 6.8$ & 0.205 & $1.9 \pm 5.8$ & 0.430 \\
BAI & $\mathbf{V}$ & $-0.9 \pm 5.3$ & 0.630 & $-2.6 \pm 5.6$ & 0.267 \\
\hline
\end{tabular}

$\boldsymbol{\nabla}$, better performance at lower scores.

BAI, Beck anxiety inventory; BDI, Beck depression inventory.

assessment that was maintained in the late-PBMT evaluation. However, in all evaluations, the means remained within the classification of mild depression. BAI self-assessment demonstrated a progressive increase in average post- $P B M T$ and late-PBMT assessments, but these levels of anxiety are classified as a minimum degree of anxiety.

\section{Discussion}

This study verified the effects of $P B M T$ on patients during the convalescence period and chronic patients, both with severe TBI, with different lesions due to TBI. The most frequent was the DAI, which is a microscopic lesion with severe consequences, high mortality, associated with temporary and permanent disabilities, evidenced up to 12 months post-trauma. ${ }^{22}$

Of the four participants who presented TBI with DAI, three had an increase in bilateral MFV MCAR. Consider that the disabilities resulting from DAI are related to rotational forces involving shear axon injury, dysregulation of $\mathrm{CBF}$, and cerebral metabolism, with direct consequences on memory functions, difficulty in learning new information, problem solving, and planning, PBMT by promoting better blood circulation that facilitates cerebral oxygenation can contribute to neurogenesis and improve brain function. ${ }^{23-27}$

The CBF and MFV MCAR and MCAL of each participant increased, although the pre- and post-PBMT average were not statistically significant. Schiffer et al. also observed improvement in CBF after an application of PBMT on 10 patients with a history of depression, anxiety, and chemical use. The PBMT $(810 \mathrm{~nm})$ was applied at two different areas of the frontal region with fluence of $60 \mathrm{~J} / \mathrm{cm}^{2}$. The researchers measured the amount of hemoglobin in the right and left prefrontal region and found an increase in local CBF in irradiated patients, although not statistically significant. ${ }^{28}$

We found a significant increase $(p \leq 0.007)$ of left PSV after $P B M T$, a result similar to Salgado et al., who applied $P B M T\left(627 \mathrm{~nm}, 70 \mathrm{~mW} / \mathrm{cm}^{2}, 10 \mathrm{~J} / \mathrm{cm}^{2}\right)$ at four points of the frontal and parietal region for $30 \mathrm{sec}$ each, twice a week for 4 weeks in 25 healthy elderly women ( $>65$ years), and they also observed a significant increase of PSV and ED of the left MCA (25\% and 30\%, respectively) and the basilar artery (between $17 \%$ and $25 \%$ ). ${ }^{11}$ We believe that the increase in PSV was caused by the shear stress in the endothelium walls, leading to an increase in local pressure, which favored the activation of signaling cascades in the endothelial cells, with consequent acute release of nitric oxide (NO) and prostacyclin. ${ }^{29,30}$ In that way, $P B M T$, by facilitating the increase of the $\mathrm{CBF}$, stimulates the production of $\mathrm{NO}$, which is an important molecule of neuronal signaling, with the capacity to provoke vasodilation from the stimulation of guanylate cyclase, which is soluble in the formation of cyclic GMP, which will activate the protein kinase G, leading to the reuptake of $\mathrm{Ca}_{2}+$ and the opening of the calciumactivated potassium channels, consequently reducing the $\mathrm{Ca}_{2}+$ concentration in the endothelium, thus promoting vasodilation. $^{31}$

In this study, the therapeutic responses were related to the period between the TBI and the beginning of the PBMT; participants were in the period from 4 months to 4 years post-TBI. CBF increased with increase of MFV MCAR and MCAL in all participants who were between 7 and 8 months post-TBI. In contrast, the only participant at 18 months postTBI had a reduction of MFV MCAR and MCA post-PBMT (MCAR: $56-42 \mathrm{~cm} / \mathrm{sec}$; MCAL: $65-58 \mathrm{~cm} / \mathrm{sec}$, respectively), suggesting a low therapeutic response for cases in whom the injury occurred $>12$ months.

Our study observed that of the three participants with a subarachnoid hemorrhage, two obtained an increase in the MFV MCAR and MCAL post-PBMT, and one presented different vasomotor behavior than expected. This participant was in the fourth month after TBI and initially the MFV MCAR was $57 \mathrm{~cm} / \mathrm{sec}$, after the $P B M T$, the MFV MCAR reduced to $32 \mathrm{~cm} / \mathrm{sec}$, which is below the established value $(<42 \mathrm{~cm} / \mathrm{sec})$ and maintenance of the MFV MCAL $(49 \mathrm{~cm} / \mathrm{sec})$, suggesting an anti-inflammatory response, with reduction of $\mathrm{CBF}$ in the hemisphere with greater impairment.

Hennessy and Hamblin argue that PBMT might have wider systemic effects. That is, it may stimulate the production of macrophages and mast cells, which can benefit the brain. ${ }^{28}$ Moreover, regardless of the area of treatment, it could lead to negative regulation of proinflammatory cytokines and to the positive regulation of anti-inflammatory cytokines throughout the body, which means that the brain can still benefit from the positive effects, even when it is not the target of the therapy.

The evaluation of cerebral hemodynamics verified the increase in CBF, which according to Ohm's law has a direct correlation with cerebral peripheral perfusion and is inversely proportional to cerebral peripheral vascular resistance. Increasing peripheral cerebral perfusion increases vascularization in adjacent tissues, thus favoring brain function. In that way, we believe that the increase in CBF in MCAR and MCAL has a positive effect on improving brain function.

Brain function is related to the roles played by the rightand left-brain hemispheres, which act as complementary parts of an integrated system. Although these hemispheres are complementary, they have distinct roles in the process of acquiring knowledge and in the conduct of humans. ${ }^{32}$

In this study, we used the neuropsychological evaluation to verify the possible neurocognitive and neurobehavioral 
changes, both mediates and late, due to PBMT. The ability for visuospatial learning is related to the medial temporal lobe in the nondominant hemisphere.

The Rey's complex figure-immediate recall evaluation (E7) exhibited a trend toward improvement $(p<0.07)$ in the average performance between the pre-PBMT and post-PBMT periods, which demonstrates the trend toward improvement in visual episodic memory, planning, organizational skills, and executive functions.

The evaluation of the symbol test, comparing pre- and post-PBMT, also showed a tendency to improve, with an increase in information processing speed. We observed a positive linear trend for executive function in the inhibition of Stroop 3. Naeser et al. ${ }^{13}$ also applied PBMT in two patients suffering from chronic TBI, who presented cognitive deficits, functional capacity losses, with serious repercussions on their lives. One of the patients was subjected to Stroop 3, and presented positive performance between the first and second evaluation. Naeser et al. also used PBMT in patients with moderate chronic neurological lesions, with significant results in Stroop 3. ${ }^{14}$

The performance during RAVLT language test in the post-PBMT and late-PBMT suggests some improvement. The individual performance of two participants increased by 1.46 in the $z$ score. Naeser et al. ${ }^{14}$ also observed improvement in verbal learning and memory assessed by California Verbal Learning Test-Second Edition (CVLT-II); after 18 sessions of $P B M T$ in 11 patients, the significance over time was found with moderate and chronic TBI. In this case, they used a device emitting red $(633 \mathrm{~nm})$ and infrared $(810 \mathrm{~nm})$ radiation with power and energy density in the order of 10 times higher than that used in our study. Hipskind et al. ${ }^{33}$ applied pulsed $P B M T$ to 12 veteran military victims of chronic TBI using 2 devices with 220 infrared LEDs and 180 LEDs emitting red radiation, generating a power of $3.3 \mathrm{~W}$ and an energy density of $7.7 \mathrm{~J} / \mathrm{cm}^{2}$. After neuropsychological evaluation, they observed a significant improvement in performance in 8 of the 12 tests performed, among them being verbal learning assessment, performed by the CVLT-II.

In our study, the participants reported a reduction in the symptoms of post-traumatic stress disorder, with a reduction in the complaint of insomnia and depression, also evidenced in the self-assessment of depression by the BDI. The mean reduction in the post- $P B M T$ remained in the late- $P B M T$ evaluation. The means for the self-evaluation of BAI anxiety levels also increased, although these levels only reduced from the minimum grade to the mild grade. In this case, due to the fact that $75 \%$ of patients use regular anxiolytics and antidepressants, the results were certainly influenced by $P B M T$ and drug therapy. Morries et al. used PBMT with high energy density ranging from 55 to $81 \mathrm{~J} / \mathrm{cm}^{2}$ in the near infrared (NIR) region in 10 patients with chronic TBI during 10 sessions for 2 months. In these conditions, they also observed reduction of chronic symptoms of TBI, such as headache, sleep disorders, cognition, mood dysregulation, anxiety, and irritability post-PBMT. ${ }^{34}$ Schiffer et al. ${ }^{28}$ also used self-assessment scales for depression and anxiety (Hamilton scale) after applying $P B M T$ session in 10 patients diagnosed with depression, anxiety, and substance abuse, and they found significant reductions in 2 weeks and remission in some patients after 6 weeks of irradiation. Saltmarche et al. used the pulsed LED with $P B M T$, in the
$810 \mathrm{~nm}, 10 \mathrm{~Hz}$ spectrum, combined with a daily intranasal device to treat patients with Alzheimer's disease with moderate-to-severe dementia for 12 weeks of active treatment with 4 weeks of rest. The evaluated patients presented improved sleep pattern and reduced incidence of rage and anxiety. ${ }^{35}$

Evidence has shown that $P B M T$ in regions of the visible and NIR provides neuroprotection and neuroregeneration. This effect can be explained by the fact that radiation when penetrating in the cranial cavity is absorbed by cytochrome $\mathrm{C}$ oxidase (CCOx), which is a terminal enzyme in the mitochondrial respiratory chain of cortical neurons, promoting the increase in cellular respiration for a time beyond exposure. Consequently, we observed the reduction of reactive oxygen species and increased production of adenosine triphosphate (ATP), which is an important nucleotide for the central nervous system and contributes to the recovery of depressive disorders. The reduction of platelet ATP has been associated with depression. ${ }^{23,36,37}$

\section{Conclusions}

The aim of this study was to evaluate hemodynamic conditions before and after PBMT in patients with TBI. At the same time, we searched prospectively for possible neurocognitive changes as a result of the use of PBMT in patients with TBI.

PBMT contributed to the increase of the CBF, evidenced mainly by the increase of the left PSV, consequently increasing the cerebral oxygenation that had repercussion in the peripheral cerebral perfusion contributing to the improvement of cerebral function.

The neuropsychological assessment showed a trend to improve visual and verbal episodic memory, planning, and processing speed, which suggests some improvement of cognitive function in these patients.

\section{Limitations}

This study had limitations, including the small sample size, absence of a sham control group, and different types of patients with severe TBI.

Although no optimal dose has been demonstrated for the treatment of TBI, since in this study the dosimetry was lower than in other studies, this may have affected behavioral and blood flow changes.

There is insufficient literature to determine whether red or NIR should be used alone or in combination in PBM treatments. NIR would penetrate deeper, and this study used only red LEDs. This should be considered a limitation, especially as the study is working with severe TBI.

More studies are needed to determine whether severe cases of TBI would need longer or more frequent treatments. Other studies could determine the response to therapy in the most severe cases.

In addition, further studies on the effects of unilateral, left-only, right, and bilateral lesions, and their effects on treatment response would be needed.

The positive results indicate the need for additional studies with a larger sample size, also using identifiable biological markers in the process of neuroregeneration. 


\section{Acknowledgments}

We thank David J. Fontoura for the statistical analysis and Prof. Luciano Queiroz-Unicamp for allowing the use of his tomographic images.

\section{Author Disclosure Statement}

No competing financial interests exist.

\section{References}

1. Menon DK, Schab K, Wright DW, Maas AI. Position statement: definition of traumatic brain injury. Arch Phys Med Rehabil 2010;91:1637-1640.

2. Canova JCM, Bueno MFR, Oliver CCD, et al. De CHM. Cranioencephalic trauma of accident victims of motorcycles. Arq Ciênc Saúde 2010;17:9-14.

3. Surano IC, Benites VM. Traumatic brain injury, IN Urgencies and emergencies. PRADO, RAMOS e VALLE, 3rd ed. São Paulo, Artes médicas, 2018.

4. Kondo K, Maruishi M, Ueno H, et al. Pathophysiology of prospective memory failure after diffuse axonal injuryLesion-symptom analysis using diffusion tensor imaging. BMC Neurosci 2010;11:147.

5. Mujica M, González G, Larraín C, Miller P, Castoldi F. Diffuse axonal endemic brain magnetic resonance. RevistaChilena de Radiologa 2003;9:182-186.

6. Santos AJS. Effects of low power laser therapy on melanoma in vitro assay. Dissertação de Mestrado-Instituto de Pesquisas Energéticas e Nucleares, IPEN-SP, 2013.

7. Lins RDAU, Dantas EM, Lucena KCR, Catão MHCV, Granville-Garcia AF, Neto 1GC. Biostimulatory effects of low power laser in the repair process. An Bras Dermatol 2010;85:849-855.

8. Corazza AAV. Fotobiomodulação Comparativa Entre Laser e LED de Baixa Intensidade na Angiogênese de Feridas Cutâneas em Ratos. Dissertação de Mestrado-Escola de Engenharia de São Carlos/Faculdade de Medicina de Ribeirão Preto/Instituto de Química de São Carlos, Universidade de São Carlos.

9. Santos MCM, Gomes Filho FC, Nicolau RA. Therapeutic Effects of Light Emitting Diode (LED) in Lactacionais Mastites. Revista Univap, São José dos Campos-SP, v. 18, n. 32, dez.2012. ISSN 2237-1753.

10. Nicolau RA, Zângaro RA. Efeito do Laser de Baixa Potência (As Gaal) em Tecido Ósseo de Rato Submetido a Lesão, Analisado por Histomorfometria Óssea. São José dos Campos, SP: Dissertação de Mestrado, Programa de Pós-Graduação em Engenharia Biomédica Univap, 2001.

11. Salgado AS, Zângaro RA, Parreira RB, Kerppers II. The effects of transcranial photobiomodulation therapy (PBMT) on cerebral blood flow in the elderly women. Lasers Med Sci 2015;30:339-346.

12. Tedford CE, Lapp S, Jacques S, Anders J. Quantitative analysis of transcranial and intraparenchymal light penetration in human cadaver brain tissue. Lasers Surg Med 2015;47:312-322.

13. Naeser MA, Saltmarche A, Krengel MH, Hamblin MR, Knight JA. Improved cognitive function after transcranial, light-emitting diode treatments in chronic, traumatic brain injury: two case reports. Photomed Laser Surg 2011;29: 351-358.

14. Naeser MA, Zafonte R, Krengel MH, et al. Significant improvements in cognitive performance post-transcranial, red/ near-infrared light-emitting diode treatments in chronic, mild traumatic brain injury: open-protocol study. J Neurotrauma 2014;31:1008-1017.

15. Hamblin MR. Shining light on the head: photobiomodulation for brain disorders Published by Elsevier B.V. This is an open access article under the CC BY license. Available at: http://creativecommons.org/licenses/by/4.0/2016

16. Radanovic M, Scaff $M$. Use of transcranial doppler for monitoring of cerebral vasospasm secondary to hemorrhage subarachnoid. Thesis presented at the Neurological Clinic of the Clinical Hospital of the Faculty USP, São Paulo-SP.

17. Poulton RG, Moffitt TE. The Rey-Osterreith complex figure test: norms for young adolescents and an examination of validity. Arch Clin Neuropsychol 1995;10:47-56.

18. Kessler BJD, Deluca JRO. Complex figure test performance following anterior communicating artery aneurysm. Arch Chm Newpsychol 1996;I:21-28.

19. Bravo MS. Standardization of the complex figure test of Rey for the elderly population of the municipality of Curitiba. Psicol Bulletin 2014;LXIII:115-127.

20. Opasso PR, Barreto SS, Ortiz KZ. Phonemic verbal fluency task in adults with high-level literacy. Einstein 2016;14:398-402.

21. Barbosa MF, Abdala N, Carrete Jr., H, et al. Conventional transcranial doppler in asymptomatic volunteers: variability and reference values for blood flow. Arq Neuropsiquiatr 2006;64:829-838.

22. Vieira RCA. Recovery of victims of axonal injury diffuse and associated factors 2015. Tese (Doutorado em Enfermagem na Saúde do Adulto)_Escola de Enfermagem, Universidade de São Paulo, São Paulo, 2015. DOI: 10.11606/T.7.2015.tde13052015-105652 (Last accessed July 25, 2018).

23. Andrade AF, Paiva WS, Amorim RLO, Figueiredo EG, Neto ER, Teixeira MJ. Mecanismos de lesão cerebral no Traumatismo crânio encefalico. Rev Assoc Med Bras 2009; 55:75-81.

24. Ianof JN, Fraga FJ, Ferreira LA, et al. Comparative analysis of the electroencephalogram in patients with Alzheimer's disease, diffuse axonal injury patients and healthy controls using LORETA analysis. Dement Neuropsychol 2017;11:176-185.

25. Xuan W, Vatansever F, Huang L, Hamblin MR. Transcranial low-level laser therapy enhances learning, memory, and neuroprogenitor cells after traumatic brain injury in mice. J Biomed Opt 2014;19:108003.

26. Wu X, Dmitriev AE, Cardoso MJ, et al. $810 \mathrm{~nm}$ Wavelength light: an effective therapy for transected or contused rat spinal cord. Lasers Surg Med 2009;41:36-41.

27. Hamblin MR. Photobiomodulation for traumatic brain injury and stroke. J Neuro Res 2018;96:731-743.

28. Schiffer F, Johnston AL, Ravichandran C, et al. Psychological benefits 2 and 4 weeks after a single treatment with near infrared light to the forehead: a pilot study of 10 patients with major depression and anxiety. Behav Brain Funct 2009;5:46.

29. Ballermann BJ, Dardik A, Eng E, Liu A. Shear stress and the endothelium. Kidney Int 1998;67:S100-S108.

30. Chistiakov DA, Orekhov AN, Bobryshev YV. Effects of shear stress on endothelial cells: go with the flow. Acta Physiol 2017;219:382-408.

31. Hennessy M, Hamblin MR. Photobiomodulation and the brain: a new paradigm. Journal of Optics 2017;19. Available at: http://iopscience.iop.org/article/10.1088/2040-8986/ 19/1/013003/meta (Last accessed June 30, 2018).

32. Freitas KN. Mental representations, visual images and knowledge in Vygotsky's thought. Ciência Cognição 2005; 06:109-112. 
33. Hipskind SG, Grover Jr., FL, Fort TR, et al. Pulsed transcranial red/near-infrared light therapy using light-emitting diodes improves cerebral blood flow and cognitive function in veterans with chronic traumatic brain injury: a case series | photomedicine and laser surgery. Photomed Laser Surg 2018 [Epub ahead of print]; DOI: 10.1089/pho .2018.4489.

34. Morries LD, Cassano P, Henderson TA. Treatments for traumatic brain injury with emphasis on transcranial nearinfrared laser phototherapy. Neuropsichiatr Dis Treat 2015; 2015:2159-2175.

35. Saltmarche AE, Naeser MA, Ho KF. Significant improvement in cognition in mild to moderately severe dementia cases treated with transcranial plus intranasal hotobiomodulation: case series report. Photomed Laser Surg 2017;35: 432-441.

36. Rojas JC, Gonzalez-Lima F. Neurological and psychological applications of transcranial lasers and LEDs. Biochem Pharmacol 2013;86:447-457.
37. Pedrazza EL, Bonnan CD. Nucleotidases and depression: effect of antidepressant drugs on extracellular ATP catabolism. Masters dissertation. Pontifical University Catholic Church of Rio Grande do Sul. 2007; Chapters 1.2, 1.3:pp. 11-12.

Address correspondence to: Ana Maria Costa Carneiro, PhD Stricto Sensu Program Master and Doctorate in Biomedical Engineering Anhembi Morumbi University São José dos Campos 12247-016 São Paulo Brasil

E-mail: amcostac@gmail.com

Received: May 19, 2019. Accepted after revision: August 6, 2019. Published online: October 3, 2019. 